*Correspondence to: Steven J. Hinder, The Surface Analysis Laboratory, Faculty of Engineering \& Physical Sciences (A1), University of Surrey, Guildford, Surrey GU2 7XH, UK.

Email: s.hinder@surrey.ac.uk Fax:+44 (0) 1483686291.

\title{
SIMS Fingerprint Analysis on Organic Substrates.
}

\author{
Steven J. Hinder ${ }^{*}$ and John F. Watts
}

The Surface Analysis Laboratory, Faculty of Engineering \& Physical Sciences (A1), University of Surrey, Guildford, Surrey GU2 7XH, UK.

\begin{abstract}
:
ToF-SIMS has been used in the analysis of latent fingerprints (dactyloscopy) with a view to use in forensic and biomedical applications. Szynkowska et al have demonstrated that SIMS can be employed to produce images of whole fingerprints on inorganic surfaces such as steel, brass and glass (1). We demonstrate the detection of a range of exogenous materials in fingerprints including industrial lubricants, personal care products and pharmaceuticals on organic surfaces. We demonstrate fingerprint image acquisition on model substrate materials such as $\mathrm{Si}$ wafer and on more 'difficult' organic substrates such as polymeric coatings, paper and printed card. SIMS images, even when acquired at low resolution, clearly delineate those regions associated with the underlying substrate materials and those from the latent fingerprint. Fingerprint images acquired by SIMS imaging are capable of visualising the minutiae associated with fingerprints with high resolution. Such minutiae are the key latent fingerprint features that must be visualised so that a forensic fingerprint examiner may make an identification.
\end{abstract}

\section{Keywords :}

Time-of-Flight Secondary Ion Mass Spectrometry; Fingerprint analysis; Fingerprint minutiae; Imaging SIMS.

\section{Running Headline :}

SIMS Fingerprint Analysis on Organic Substrates 


\section{Introduction :}

The surface of human skin is covered with many natural substances secreted from the eccrine, apocrine and sebaceous glands. The major secretion from these glands is water; however, both inorganic and organic substances are also secreted. It is these secretions that form the basis of much of the material left in a latent fingerprint. Dactylocopy is the branch of Forensic Science dealing with the examination of skin ridge impressions, but mainly latent fingerprints, for the purpose of identifying individuals. Currently the majority of the techniques developed to analyse latent fingerprints, such as dusting with powders or fuming cyanoacrylates, requires that a chemical or adhesive reaction takes place between the substances present in the fingerprint and the applied reagent. Typically these techniques allow the latent fingerprint to be visualised and an image captured for the purposes of analysis and identification. However, such techniques suffer from the inevitable drawback that much of the potential chemical data present in the fingerprint is lost or compromised. The use of static SIMS provides us with the opportunity to both visualise the latent fingerprint and to concomitantly acquire high resolution mass spectra of the latent fingerprint from which chemical information may be extracted.

Here, ToF-SIMS has been employed in the analysis of latent fingerprints on organic substrates with a view to its use in forensic (and possibly biomedical) applications. Szynkowska et al have demonstrated that SIMS can be employed to produce images of whole fingerprints on inorganic surfaces such as steel, brass and glass (1). Here we are interested in utilising the mass spectra resulting from fingerprint image 
acquisition as a means to identify the chemistry involved in the fingerprint material. We demonstrate the detection of a range of exogenous materials in fingerprints including industrial lubricants, personal care products and inorganic compounds. Although latent fingerprints acquired on model substrate materials such as $\mathrm{Si}$ wafer and $\mathrm{Al}$ foil are demonstrated, latent fingerprint images were also acquired on more 'difficult' organic substrates such as a PVdF/acrylic coating, paper and clingfilm.

\section{Experimental :}

\section{$\underline{\text { ToF-SIMS. }}$}

ToF-SIMS analyses of the latent fingerprints were carried out on an ION-TOF GmbH (Münster, Germany) TOF.SIMS 5 instrument. A $25 \mathrm{keV} \mathrm{Bi}_{3}{ }^{+}$primary ion beam delivering $0.35 \mathrm{pA}$ of current was employed. Images were acquired at $128 \times 128$ resolution in the MacroRaster mode of operation. The 128 resolution was selected as it provides good image resolution within an acceptable timeframe i.e. $2+$ hours per image acquisition. Superior quality images can be acquired employing higher resolutions but these require significantly longer acquisition times. Image data was acquired using 256 cycles per pixel point with 1 scan per pixel. A cycle time of 100 $\mu$ s was employed.

When producing doped fingerprints $\sim 0.5 \mathrm{~mL}$ of dopant i.e. PCP, was applied to the 'little' finger and rubbed into the skin employing the thumb. The finger tip was then allowed to 'dry out' for at least 5 minutes before the finger was lightly pressed onto the substrate of choice. 


\section{Results and Discussion:}

SIMS images, even when such images are acquired at low to moderate resolution, can clearly delineate those regions of the specimen associated with the underlying substrate material and those arising from a latent fingerprint. In Figure 1 a SIMS image of a latent fingerprint (from a finger treated with a personal care product $(\mathrm{PCP})$ ) on an organic fluorocarbon based coating is presented. The red regions observed in Figure 1 contrast the intensity of the $181 \mathrm{u}$ ion (tentatively assigned to $\mathrm{C}_{13} \mathrm{H}_{9} \mathrm{O}^{+}$) over a $1 \mathrm{~cm}^{2}$ region of the sample surface. Independent mass spectral analysis of the PCP, polymeric coating and 'clean' non-doped fingerprints (data not shown) indicates the $181 \mathrm{u}$ ion is only observed in the PCP mass spectrum. It is clearly observed in Figure 1 that the $181 \mathrm{u}$ ion is confined to those regions of the sample surface associated with the latent fingerprint friction ridges. The green regions in Figure 1 contrast the $133 \mathrm{u}$ ion $\left(\mathrm{C}_{3} \mathrm{H}_{2} \mathrm{~F}_{5}{ }^{+}\right.$fragment ion) originating from the poly(vinylidene difluoride) component of the polymeric coating employed as the substrate in this analysis. It is observed in Figure 1 that the green regions are significantly narrower than the red friction ridge regions observed in Figure 1. Typically we find that individual fingerprint friction ridges are 200-400 $\mu \mathrm{m}$ in diameter while friction ridge spacing is $\sim 100-200 \mu \mathrm{m}$. The ToF-SIMS image in Figure 1 demonstrates that SIMS is clearly able to delineate between the friction ridges features and substrate associated with a latent fingerprint. 
SIMS mass spectra obtained from whole fingerprints, detailed fingerprint regions or even individual friction ridges often yield similar mass spectra. This is advantageous when chemical analysis of the fingerprint material is required as 'real world' samples are often not ideally flat and SIMS may only be able to analyse a limited number of friction ridge features. However, for an analysis or imaging technique to be of use for dactyloscopic purposes it must be capable of visualising the minutiae (features associated with individual friction ridges) associated with latent fingerprints with good resolution. It is these minutiae that are the key latent fingerprint features required by a forensic fingerprint examiner to make an identification from a latent fingerprint. Minutiae such as bifurcations, terminals, dots, short ridges and enclosures are readily observed in the mass selected ToF-SIMS images in Figure 2. The SIMS images in Figure 2 were acquired at $128 \times 128$ resolution over a $25 \mathrm{~mm}^{2}$ region, thus the images are composed of $16,384,39 \times 39 \mathrm{um}^{2}$ pixels. Even at the moderately low pixel resolution and relatively large pixel size in Figure 2, fingerprint minutiae are readily observed employing ToF-SIMS imaging.

We have investigated fingerprints on both 'easy' model substrates such as Al foil and Si wafer and more 'difficult' substrates such as paper, cardboard and polymeric coatings (see Figure 1) with varying degrees of success (see Table 1.). Additionally we have analysed 'clean' fingerprints and those 'treated' with inorganic materials and organic compounds such as industrial lubricants and personal care products (see Figure 1). 'Clean' fingerprints typically contain element from perspiration i.e. $\mathrm{Na}, \mathrm{K}$ and $\mathrm{Cl}$ and fatty acid residues, one of the most common being squalene $\left(\mathrm{C}_{30} \mathrm{H}_{50}\right.$, m.w. 
410.39, see Schematic 1). Jickells and her colleagues have demonstrated that squalene oxidation can be use to track fingerprint aging (2).

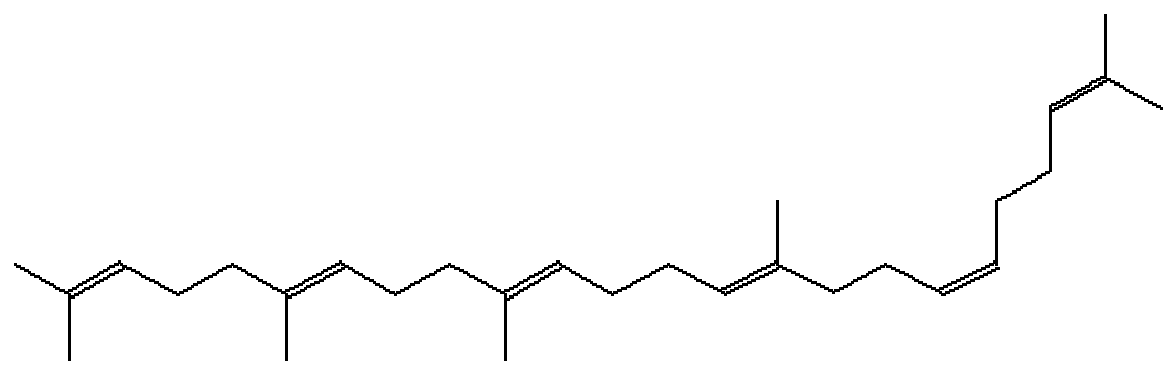

Schematic 1 . The structure of squalene.

ToF-SIMS analysis of latent fingerprints bestows the added advantage that no processing of the fingerprint or substrate is required prior to analysis. The central image in Figure 3 is that that would be expected if this sample was processed by a forensic scientist in a normal manner to visualise the fingerprint materials. In this sample two fingerprints, one from a PCP and the other a fluorinated inorganic powder have been overlaid on a polyester/polyurethane based polymeric coating. Although the outer ridges of the individual fingerprints are observed in the central image of Figure 3 the majority of the fingerprints are difficult to visualise due to their overlapping. However, using SIMS mass selected images for ions specific to each of the sample materials used to form the fingerprints, it is possible to extract latent fingerprint images of the individual fingerprints and thus eliminate the problems caused by the overlap. In Figure 3 the left hand image is extracted from the ion at 97 $\mathrm{u}$ originating from the PCP while the right hand image is extracted from the $\mathrm{F}$ ion at $19 \mathrm{u}$ which originates from the inorganic powder. The mass selected images in Figure 
3 possess very good ridge detail even though the data was only acquired at a resolution of $128 \times 128$ pixels.

The ToF-SIMS mass selected images presented in Figures $1 \& 3$ were acquired on what might be deemed idealised or model organic substrates in that the coatings employed were applied to sheet aluminium and their formulations contain components included to provide the coatings with flat, well defined surfaces i.e. flow aids. In Figure 4 a number of ToF-SIMS mass selected images acquired on substrates known to be 'difficult', forensically speaking, are presented. In Figure 4a a 2 colour overlay of a PCP fingerprint (in green) on a supermarket carrier bag (in red) is shown. The PCP fingerprint ridge detail is clearly observed, while the carrier bag substrate is clearly delineated. Indeed the carrier bag region of the image in Figure 4a clearly shows evidence of the substrates surface topography. In Figure $4 \mathrm{~b}$ a more complete PCP containing latent fingerprint image on white photocopier paper is presented. Although the photocopier paper is fibrous in nature at the area $\left(1.5 \times 1.5 \mathrm{~mm}^{2}\right)$ and pixel size $\left(\sim 120 \times 120 \mu \mathrm{m}^{2}\right)$ employed the nature of the substrate does not affect greatly the quality of the latent fingerprint image. In Figure 4c printed newspaper has had a PCP fingerprint applied to it such that the fingerprint sits astride both white typeset (right hand side) and coloured print (left hand side) regions of the newspaper. It is clearly observed in Figure $4 c$ that the clarity of the fingerprint detail may be influenced by the nature of the underlying substrate with in this case the white typeset region of the specimen providing superior and more intense fingerprint detail than the colour print region of the specimen. Employing ToF-SIMS imaging we were able to 
obtain excellent resolution mass selected images on a particularly difficult organic substrate, cling-film, as presented in Figure 4d. A latent fingerprint containing traces of an industrial lubricant was applied to cling-film, and then the cling-film was 'teased' out and stretched onto the SIMS instruments sample platen to make it as flat as possible. Indeed the very nature of the material made it amenable to this procedure allowing a very flat specimen to be obtained. Figure $4 d$ possesses very good fingerprint ridge (in green) definition and the delineation between latent fingerprint and cling-film substrate is excellent with fingerprint minutiae features being readily observed.

\section{Conclusions :}

We have demonstrated that ToF-SIMS imaging of latent fingerprints can be performed on contrasting organic substrates. We have also demonstrated that SIMS imaging of fingerprints provides sufficient ridge and fingerprint minutiae detail to allow its use for forensic identification purposes. ToF-SIMS imaging of latent fingerprints also provides mass spectrometric data concerning the chemical composition of the fingerprint. These findings may allow fingerprint mass spectrometric data to be used in forensic applications.

\section{References :}

1) Szynowska M.I, Czerski K, Grams J, Paryjczak T, Parczewski A. The Imaging Science Journal, 2007; $55: 180$.

2) Archer N, Charles Y, Elliott J, Jickells S. Forensic Sci. Int. 2005; $154: 224$. 


\section{Acknowledgements :}

The authors thank Becker Industrial Coatings for the provision of the PVdF and polyester/polyurethane coatings. 


\begin{tabular}{|l|l|}
\hline Substrate & \multicolumn{1}{c|}{ Comment on SIMS Imaging Effectiveness } \\
\hline PVdF Coating & High quality fingerprint image obtained. \\
\hline Si Wafer & Very high quality fingerprint images are readily obtained. \\
\hline Carrier Bag & $\begin{array}{l}\text { Acceptable quality fingerprint image obtained however it is } \\
\text { difficult to maintain the flatness of the substrate material. }\end{array}$ \\
\hline Photocopier paper & $\begin{array}{l}\text { Acceptable quality fingerprint image obtained however surface } \\
\text { roughness and fingerprint material adsorption into the substrate } \\
\text { is believed to lead to some loss of fingerprint image resolution. }\end{array}$ \\
\hline Newspaper & $\begin{array}{l}\text { Poor quality fingerprint image due to surface roughness and } \\
\text { changes in the nature of the substrate material affecting } \\
\text { fingerprint image resolution. }\end{array}$ \\
\hline Cling-film & $\begin{array}{l}\text { High quality fingerprint image obtained once the substrate } \\
\text { material is flattened. }\end{array}$ \\
\hline
\end{tabular}

Table 1. Author's comments on the effectiveness of SIMS imaging of latent fingerprints on the different substrates employed. 


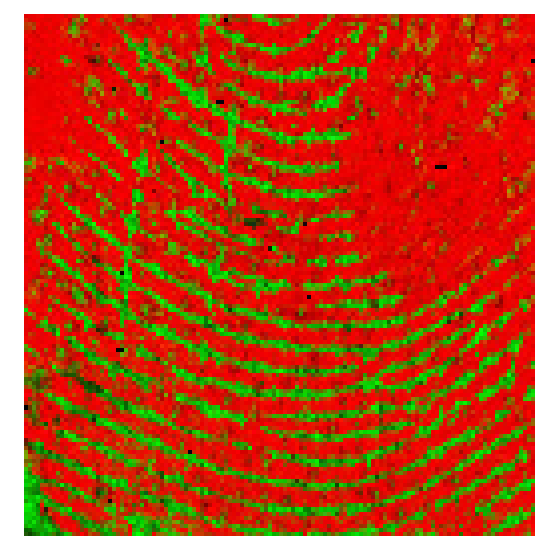

Figure 1. A $1 \mathrm{~cm}^{2}$ mass selected ToF-SIMS image presented as a 2 colour overlay image. The regions in red are from a latent fingerprint while those in green are from the underlying substrate. 


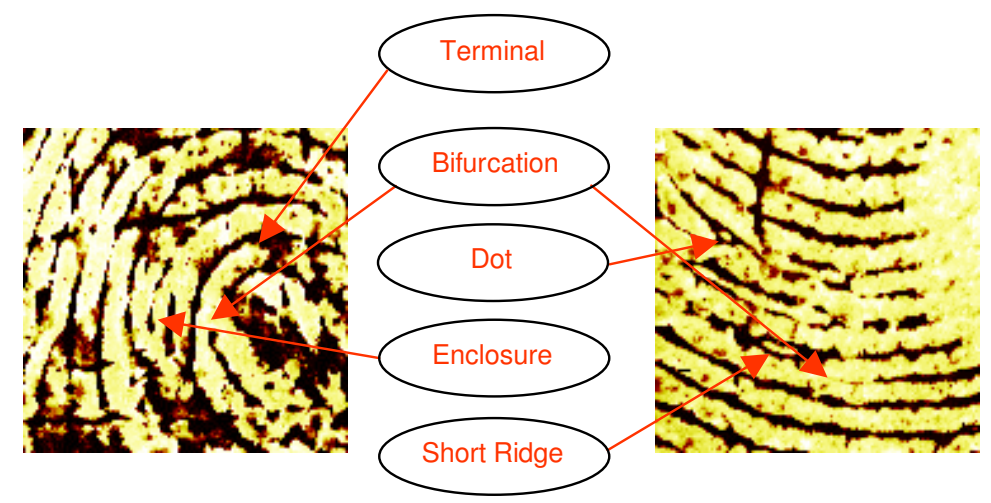

Figure 2. $25 \mathrm{~mm}^{2}$ mass selected ToF-SIMS images showing the minutiae associated with individual friction ridges in latent fingerprints. 

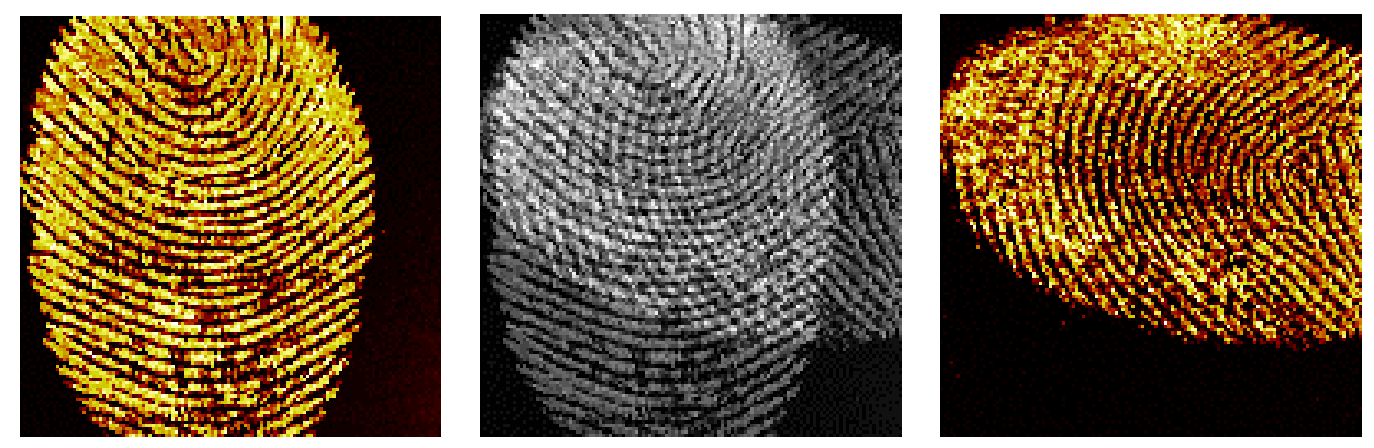

Figure $3.15 \times 15 \mathrm{~mm}^{2}, 128 \times 128$ resolution ToF-SIMS negative ion images of two overlaid finger prints on a polyester/polyurethane primer coating. 


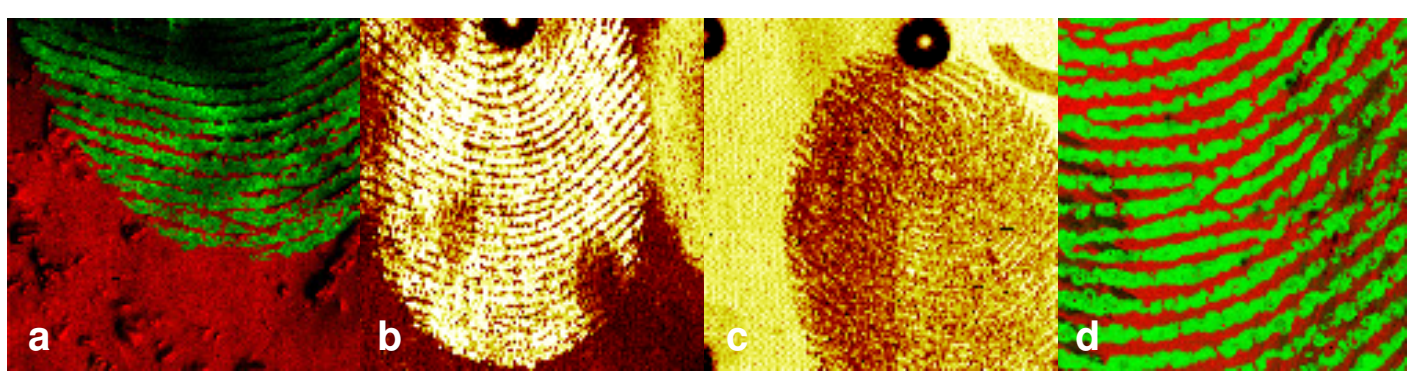

Figure 4. A montage of ToF-SIMS mass selected latent fingerprint images from a number of organic substrates, a) PCP on supermarket carrier bag, b) hand cream on white photocopier paper, c) PCP on printed newspaper and d) industrial lubricant on cling-film. 